\title{
Dramatyping: A generic algorithm for detecting reasonable temporal correlations between drug administration and lab value alterations
}

Axel Newe

According to the World Health Organization, one of the criteria for the standardized assessment of case causality in adverse drug reactions is the temporal relationship between the intake of a drug and the occurrence of a reaction or a laboratory test abnormality. This article presents and describes an algorithm for the detection of a reasonable temporal correlation between the administration of a drug and the alteration of a laboratory value course. The algorithm is designed to process normalized lab values and is therefore universally applicable. It has a sensitivity of 0.932 for the detection of lab value courses that show changes in temporal correlation with the administration of a drug and it has a specificity of 0.967 for the detection of lab value courses that show no changes. Therefore the algorithm is appropriate to screen the data of electronic health records and to support human experts in revealing adverse drug reactions. A reference implementation in Python programming language is available. 


\section{Author \& Affiliation}

2 1. Corresponding author:

3 Name: Axel Newe

4 Affiliation: Chair of Medical Informatics, Friedrich-Alexander-University Erlangen-Nuremberg, Erlangen, Germany

5 Postal address: Lehrstuhl für Medizinische Informatik, Am Wetterkreuz 13, 91058, Erlangen, Germany

6 E-mail address: axel.newe@fau.de

7 


\section{Introduction}

Harmful reactions to pharmaceutical products (Adverse Drug Reactions, ADRs) are a wellknown cause of morbidity and mortality and are one of the common causes of death in many countries (WHO, 2008; Meier et al., 2015). Even if a drug is administered correctly (i.e., correct indication, correct dose, no contraindication etc.), it still can have unwanted side effects, of which some can be harmful (WHO, 2008). Therefore, the effects of a drug therapy cannot be predicted with absolute certainty: all drugs have both benefits and the potential for harm (WHO, 2008).

Apart from the individual fate of the affected patients, ADRs are also a large economic burden because of the resources which are required for both the diagnostics and the treatment of the symptoms as well as the diseases caused by ADRs (Stausberg, 2014). ADR-related costs (e.g., for hospitalization, surgery or lost productivity) exceed the cost of the medications in some countries (WHO, 2008). Recent figures show that the situation in different countries is roughly comparable. The average cost caused by a single ADR was calculated US\$ 3420 for the USA (Hug et al., 2012), US\$ 3681 for Germany (Meier et al., 2015) and US\$ 115 for India (Rajakannan et al., 2012). This corresponds to 42\% (USA), 85\% (Germany) and 91\% (India) of the respective overall per capita expenditure on health (WHO, 2013).

The detection, assessment, monitoring, and prevention of drug-related adverse effects is termed pharmacovigilance (WHO, 2008). The general standard and still the most commonly used method of ADR detection is mandatory spontaneous reporting (Neubert et al., 2013). In the USA, the primary source of such reports is the FDA Adverse Event Reporting System (FAERS) database (FDA, 2014) of the U.S. Food and Drug Administration (FDA). Other countries have similar systems installed (BfArM, 2015). This approach, however, is prone to several problems, such as under-reporting and bias (e.g., influences by media coverage) (Neubert et al., 2013). Up to $90 \%$ of adverse events remain undetected in hospital settings, while up to one third of hospital admissions is accompanied by ADRs (Classen et al., 2011). Another approach to the discovery of ADRs is the manual chart review - the gold standard in pharmacoepidemiology. It is very precise but also time-consuming and it causes high staff expenses (Neubert et al., 2013).

An alternative approach is the analysis of Electronic Health Records (EHRs). One example is the Sentinel Initiative (FDA, 2008) of the FDA, which was launched in 2008 and which aims at the development and the implementation of a system which uses existing healthcare databases (including EHRs) to assess the safety of drugs and other medical products. Although research has been conducted in this area for years, detecting ADRs in clinical records remains a 
challenging problem (Liu et al., 2012). Typical problems are inaccurate data, incomplete patient stories, data transformation and the use of narrative text instead of coded data (Hersh et al., 2013).

However, laboratory data have been identified as suitable parameters for the detection of ADRs (Grönroos et al., 1995; Ramirez et al., 2010; Neubert et al., 2013). Reference material covering the influence of drug intake on laboratory test results is available in abundance (e.g., (Young et al., 1972; Siest, 1980; Young, 2000)). One of the criteria defined by the World Health Organization (WHO) for a standardized case causality assessment of ADRs considers the temporal relationship between drug intake and the occurrence of a reaction or a laboratory test abnormality (WHO, 2005).

This article presents a heuristic screening algorithm for the detection of reasonable temporal correlations between drug administration and lab value alterations in appropriate data. It can be parameterized and thus be adjusted to specific use cases. A reference implementation in the Python programming language is available.

\section{Background and Related Work}

\section{Secondary Use of EHR Data - Dramatyping}

Secondary Use, i.e., the use of data collected for other purposes is one of the most important fields of medical informatics research. A subdomain of this research is phenotyping: the identification and determination of observable characteristics of data sets, or of the underlying individuals on the basis of data that for example have been stored in EHRs. The analysis of EHR data already offers many opportunities to carry out phenotyping; however, the current methods still leave much room for improvement (Hripcsak \& Albers, 2013).

Difficulties arise from a peculiarity of ADRs: even in the same individual, ADRs are neither reliably reproducible nor reliably detectable (Kramer, 1981). While patients are usually treated with a drug until the desired effect occurs, an adverse reaction may show up during one treatment episode while another episode stays free of ADRs. This may be because of a set of confounders that are possibly hidden from the observer. Furthermore, the imperfect quality of data found in EHRs (as mentioned above) is a factor to be considered. Therefore, the term "phenotype" is not enough in this case - instead, the term "dramatype" (introduced by (Russell \& Burch, 1959)) should be chosen instead, because "phenotyping" refers to generally observable characteristics, whereas "dramatyping" refers to intra-individual characteristics and/or shorter observation periods. 


\section{Interactions between Drugs and Lab Values}

One subdomain of dramatyping is the detection of Drug-Lab Value Interactions (DLVI). These interactions can be caused in vivo (through the drug's influence on metabolic processes or by tissue damage) or in vitro (by the drug's influence on chemical processes during the actual laboratory tests) (Singh, Hebert \& Gault, 1972; Sonntag \& Scholer, 2001).

The most obvious source of evidence for DLVI is reference literature (like (Young, 2000)), as well as relevant publications (like (Young et al., 1972; Siest, 1980; Tatonetti et al., 2011) and many others). In addition, the individual prescribing information and drug monographs provide valuable information. The latter are usually structured, but in large parts written in free text; therefore, they can only be processed indirectly by means of computers (e.g., through Natural Language Processing, NLP). Furthermore, often no direct DLVI can be derived from the drug monographs; it requires, for example, implicit medical knowledge to infer that a listed "liver injury" is related to liver-specific laboratory parameters such as Aspartate Transaminase, Alanine Transaminase or Gamma-glutamyl Transferase. This transfer work has been done and published, for example, by (Neubert et al., 2013).

\section{Detection of Temporal Correlations}

Most approaches of mining EHR databases for drug safety signals are based on measures that quantify the degree of deviation of an observed rate of a drug-related event from a control group (Harpaz et al., 2010), like, e.g., the Relative Reporting Ratio (Hauben et al., 2005). However, in order to calculate a ratio, numerator and denominator must be known, i.e., the number of observed cases and the number of expected cases must be quantifiable. Especially the former number can be difficult to figure out since the relevant cases must be documented or at least be identifiable.

DLVIs have been proven to be suitable manifestations of such drug-related events (Grönroos et al., 1995; Ramirez et al., 2010; Neubert et al., 2013) and the temporal correlation between the occurrence of a lab value alteration and the preceding application of a drug is one of the criteria for a standardized case causality assessment of ADRs defined by the WHO (WHO, 2005) as well as by other authors (e.g., (Lucas \& Colley, 1992)).

When exactly, however, such a temporal relationship exists (and how exactly it must be manifested), is not defined unequivocally - not even nearly. There are multiple degrees of freedom:

- The overall timeframe can range between immediate reactions that are obvious and late events that become evident months after drug intake (like, e.g., the thalidomide tragedy 
106

107

108

109

110

111

112

113

114

115

116

117

118

119

120

121

122

123

124

125

126

127

128

129

130

131

132

133

134

135

136

137

138

of the late 1950s/early 1960s (Curran, 1971)).

- The lab value course itself can show various types of changes (shifts of the mean value, deviations upwards and downwards, changes of curviness...).

- The amplitude of the change is highly various as well: some liver and heart enzymes can rise tenfold or more, while other parameters like sodium or potassium only alternate in a very narrow interval, even in pathological conditions.

Since no commonly accepted definition exists, only human expert assessments of pertinent lab value courses can be referenced as gold standard. A study covering this field of research which includes an open data corpus of such assessments made by human experts was published by (Newe et al., 2015). An algorithm that aims to detect generic temporal correlations between the application of drugs and corresponding lab value changes, however, has - to best knowledge - not been published before.

\section{Confusion of Definitions}

Due to the manifold settings in pharmacovigilance, literature regarding effects of drugs is characterized by a confusion of definitions. A 2012 review (Pintor-Mármol et al., 2012) has identified 189 associated terms used in relevant publications. For the term "Adverse Drug Event" (ADE), 15 different definitions were found, for "Adverse Drug Reaction" (ADR) there were 11. This confirms the findings of a 2005 review (Yu, Nation \& Dooley, 2005), in which 10 $(A D E)$ and 11 (ADR) definitions were identified, and it shows that the situation has not improved in the intermediate years.

Thus, for the rest of this article, the usual keywords or abbreviations will not be used in association with the presented algorithm. Instead, the new term "Observable Drug Event" (ODE), which - to best knowledge - has never been used before, will be used. In accordance with the criteria of the World Health Organization for a standardized case causality assessment of ADRs (WHO, 2005) and in analogy to the definitions in (Lucas \& Colley, 1992), an "Observable Drug Event" shall be defined as an "event or laboratory test alteration with reasonable time relationship to drug intake". This definition intentionally excludes the harmfulness or desirability of the observed events or laboratory test alterations (i.e., desired events or lab value alterations are included). This definition also excludes all other external factors or possible confounders and considers only the currently observed events (i.e., the lab value changes or any other event) and the medication intake. Furthermore, this definition intentionally includes all types of relationships, including correlations as well as causal relationships. 
139

140

141

142

143

144

145

146

147

148

149

150

151

152

153

154

155

156

157

158

159

160

161

162

163

164

165

166

167

168

169

170

\section{Methods}

\section{Ethics}

For the development of the algorithm described below, only previously published and freely available data has been used. Human subjects were not involved during any stage of the presented research.

\section{Development of the Algorithm}

As outlined above, the timeframe in which DLVI can be observed ranges between immediate reactions and late events that occur months after drug intake. These two extremes are out of focus of this article: immediate reactions are usually obvious and long term manifestations are hard to detect by means of EHRs. Therefore, the algorithm for detecting temporal correlations between drug administration and lab value alterations described here focuses on short term reactions, since they are well suited to be unveiled by EHR analysis (Newe et al., 2015).

Due to the lack of an unambigious definition of "temporal correlations", the 400 curves and their assessment by human experts published in (Newe et al., 2015) were taken as reference and gold standard for the development of the algorithm. Since using only a minority of this limited data for the development would not be reasonable (Hawkins, 2004), the Ground Truth data set was not divided into training data and validation data. Instead, an external dataset was used for validation (see next section). The curves and the corresponding expert assessments were analyzed by means of feature engineering in order to inductively work out the necessary classfication process.

Since a remarkable number (47 out of $400 ; 11.75 \%$ ) of these curves was classified as "no assessment possible" by the human experts, the algorithm has been designed in a way that it produces results in a three-part nominal classification scale as well:

- "temporal correlation" if a reasonable temporal correlation between drug intake and lab value alteration is detected;

- "no change" if no correlation between drug intake and lab value alteration exists, and

- "no assessment" if the data basis does not allow for an assessment or if the incertainty is too high.

In order to avoid issues regarding the diversity of reference intervals of lab parameters (which might arise, e.g., from patient parameters like age, sex etc. or from individual laboratory equipment), the algorithm has been designed in a way that it expects normalized lab values (as proposed in formula (1) of (Newe et al., 2015), see Table 1) as input. 
171 Table 1: Formula for normalizing lab values as published in (Newe et al., 2015).

\begin{tabular}{|l|l|}
\hline Formula & $L V_{n}=\frac{L V_{a}-B V_{l}}{B V_{u}-B V_{l}}$ \\
\hline Parameters & $\begin{array}{l}\mathbf{L V}_{\mathrm{n}} \text { is the normalized lab value, } \mathbf{L V}_{\mathrm{a}} \text { is the actual (absolute) lab value, } \mathbf{B V}_{\mathbf{l}} \text { is the patient-specific } \\
\text { lower border value and } \mathbf{B V}_{\mathbf{u}} \text { is the patient-specific upper border value. }\end{array}$ \\
\hline
\end{tabular}

172 The output format has also been specified to match the proposal in (Newe et al., 2015) 173 (Figure 1).

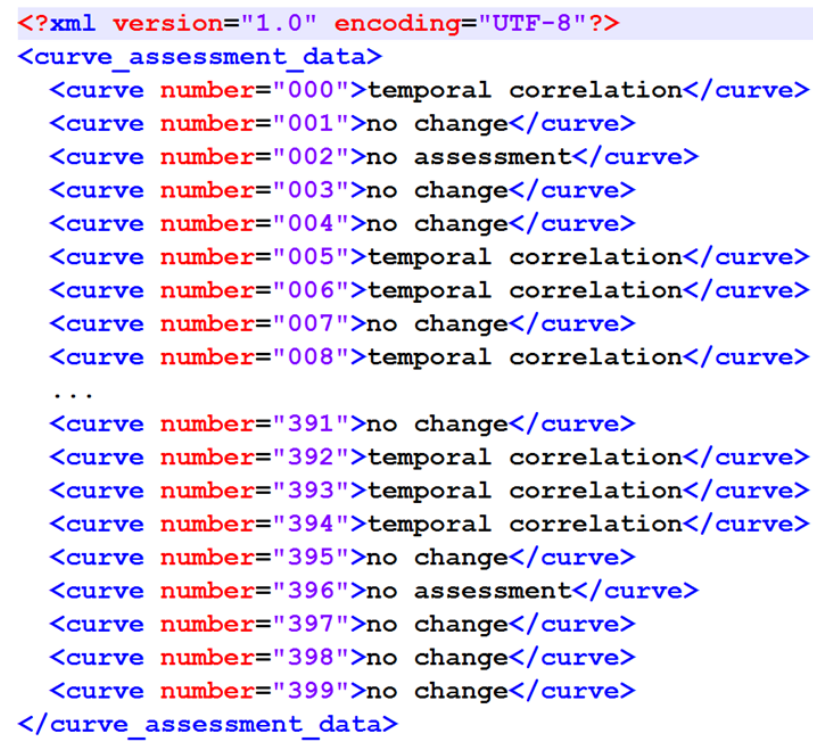

175 Figure 1: XML structure of the output XML. It matches the original proposal from (Newe et al., 2015).

176 Finally, some quality objectives have been specified. In (Newe et al., 2015), a Concordance 177 Score $S_{c}$ was introduced in order to provide a simple numerical value as a means for comparing 178 external (e.g., algorithmic) assessment results of the Ground Truth data corpus with the 179 assessment made by human experts. The lowest $S_{c}$ achieved by a human expert in (Newe et al., 180 2015) was 0.766 and therefore this value has been set as the minimum to be achieved by the 181 algorithm. Furthermore, the worst decision that the algorithm could make was assumed to be a 182 "no change" classification where actually a temporal correlation exists. Therefore, the 183 specificity for the "no change" classification to be achieved by the algorithm has been specified 184 to be 0.85 or better. Finally, since the algorithm was designed to detect temporal correlations, 185 the sensitivity for the "temporal correlation" classification to be achieved by the algorithm has 186 also been specified to be 0.80 or better. The latter two specifications, however, have not been 187 considered mandatory. 


\begin{tabular}{|l|l|}
\hline ID & Requirement \\
\hline 1 & $\begin{array}{l}\text { The algorithm shall classify the course of a lab value curve according to the existence of a temporal } \\
\text { relationship between a change of this lab value curve and the administration of a drug. }\end{array}$ \\
\hline 1.1 & $\begin{array}{l}\text { The algorithm shall classify each lab value / medication episode into one of three categories: "temporal } \\
\text { correlation", "no change" or "no assessment". }\end{array}$ \\
\hline 1.2 & The algorithm shall use normalized lab values (according to formula (1) in (Newe et al., 2015)) as input. \\
\hline 1.3 & The algorithm shall use the days of drug administration as input. \\
\hline 2 & The algorithm shall write the results into an XML file as modeled in Figure 1. \\
\hline 3 & The Concordance Score $\mathrm{S}_{\mathrm{c}}$ of the algorithm result shall be larger than 0.766. \\
\hline 4 & The algorithm should achieve a specifity for the "no change" classification of $>0.85$. \\
\hline 5 & The algorithm should achieve a sensitivity for the "temporal classification" of $>0.80$. \\
\hline
\end{tabular}

A summary of all requirement specifications for the devlopment of the algorithm is listed in Table 2.

The reference implementation was developed using WinPython (http://winpython.github.io/) version 2.7.10.2.

\section{Validation of the Algorithm}

The validation of the algorithm was carried out in two steps in order to verify both the retrodictive value and the predictive value: First, the concordance of the algorithm classifications with the classifications made by human experts was verified, and second, the non-existence of overfitting was verified.

While the algorithm has been developed by means of feature-engineering (i.e., induction) from the data corpus published in (Newe et al., 2015), the first step of the validation was carried out by means of deduction. Therefore, all curves from that data corpus were assessed by the algorithm and the classification results were then compared to the reference classifications that have been determined by human experts. By running the algorithm with varying parameters, it has been calibrated to the best possible outcome.

This result comparison was carried out by means of the DOG software application ("Data Observation Gadget") published with the data corpus article, using the result file created by the algorithm (Figure 1) as the input and the Concordance Score $S_{c}$ as recommended the as output. In addition to that, a detailed breakdown of the classification results was created.

In order to ensure that the algorithm is not biased by overfitting, a validation dataset was assessed by the algorithm and the result of this assessment was compared to the result of the assessment of the Ground Truth data corpus. The criterion used for the comparison was the number of data sets that are classified by a certain step of the algorithm. The validation data consisted of the remaining 502 episodes of drug administrations with temporarily 
213 corresponding lab value observations that had not been sampled for the Ground Truth data 214 corpus (see (Newe et al., 2015) for details).

\section{$215 \quad$ Results}

\section{The Algorithm}

217 The algorithm uses signal processing methods and therefore treats the lab value curve as a discrete-time biosignal. The terms needed to understand the following description of the algorithm are defined in Table 3.

Table 3: Terms used for the description of the algorithm.

\begin{tabular}{|l|l|}
\hline Term & Explanation \\
\hline lab value curve & The normalized laboratory values of one medication episode. \\
\hline pre-phase & The time period before the first application of the drug. \\
\hline mid-phase & $\begin{array}{l}\text { The time period from the first application of the drug until the last application of } \\
\text { the drug. }\end{array}$ \\
\hline post-phase & The time period after the last application the drug. \\
\hline fitted curve & $\begin{array}{l}\text { An artificially generated signal curve that has been fitted to the lab value curve } \\
\text { (i.e., parameters like slope and intercept of the fitted curve have been optimized } \\
\text { to match the lab value curve by means of the Levenberg-Marquardt algorithm } \\
\text { (Moré, 1978)). }\end{array}$ \\
\hline low-pass filtered data & $\begin{array}{l}\text { The lab value curve after application of a one-dimensional Gaussian low-pass filter } \\
\text { (Haddad \& Akansu, 1991) ( } \sigma=1.5 \text { ). }\end{array}$ \\
\hline removed outliers & $\begin{array}{l}\text { The lab value curve after replacement of the two most extremely deviating values } \\
\text { by the median. }\end{array}$ \\
\hline
\end{tabular}

221 The algorithm (Figure 2A) is generally divided into two major components: a main loop and a post-processing chain. An optional pre-processing step which checks if lab values are within the reference range [0..1] can be activated (a positive check would result in "no change"), but is deactivated by default in the reference implementation. Each step is only performed if the previous step ended without a classification result.

The main loop consists of four steps (Figure 2B):

- Main Loop Step 1: Checks if the lab value curve is constant during the pre-phase (i.e., if the values derive less than a parameterizable tolerance from a fitted constant curve) but no longer in the mid-phase/post-phase. If this is true, it checks if the mean values of the pre-phase differ from the mean values of the mid-/post phase (i.e., if the prephase mean values derive more than a parameterizable tolerance from the mean values of later phases). If this is also true, this step results in "temporal correlation". 
233

234

235

236

237

238

239

240

241

242

243

244

245

246

247

248

249

250

251

252

253

254

255

256

257

258

259

260

261

262

263

264

- Main Loop Step 2: Checks if the lab value curve is linear (i.e., if the values derive less than a defined parameterizable threshold from a fitted linear curve). A positive check results in "no change".

- Main Loop Step 3: Checks if the lab value curve is linear for mid-phase and post-phase (i.e., if the values derive less than a parameterizable threshold from a fitted linear curve). A positive check results in "no change".

- Main Loop Step 4: Checks if the mean values of the pre-phase differ from the mean values of the mid-/post phase (as in Main Loop Step 1). A positive check results in "temporal correlation".

This main loop is executed three times:

- with the original, unfiltered data in the first pass (Algorithm Step 1),

- $\quad$ with low-pass filtered data in the second pass (Algorithm Step 2), and

- with removed outliers and low-pass filtered data in the third pass (Algorithm Step 3).

The post-processing chain (Figure $2 \mathrm{C}$ ) comprises four additional steps:

- Algorithm Step 4: Checks low-pass filtered data for differences in slope between the phases (i.e., checks if the low-pass filtered lab values are rising or falling in the prephase but not in the mid-phase or the post-phase). If the pre-phase has a falling edge or rising edge, but the mid-phase and the post-phase has not, the result is "no change". If the pre-phase has a rising or falling edge, and either mid-phase or the post-phase does have a rising or falling edge as well, the result is "no assessment".

- Algorithm Step 5: Checks lab values with removed outliers for deviations from the mean value (i.e., checks if the lab values with removed outliers differ more than a parameterizable tolerance from their mean value). A negative check results in "no change".

- Algorithm Step 6: Checks if lab values are alternating strongly (i.e., if they do "zigzag"). A positive check results in "no assessment".

- All lab value curves that could not be classified by the previous steps are finally classified as "temporal correlation".

Some of the steps seem to be redundant, but it must be kept in mind that a dataset that could be tagged with a result drops out of further processing. Therefore, the sequential processing of the steps is essential. 


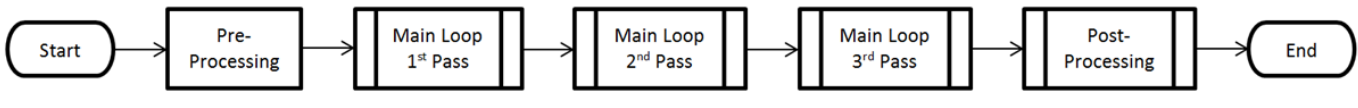

(B)

Main Loop Flowchart

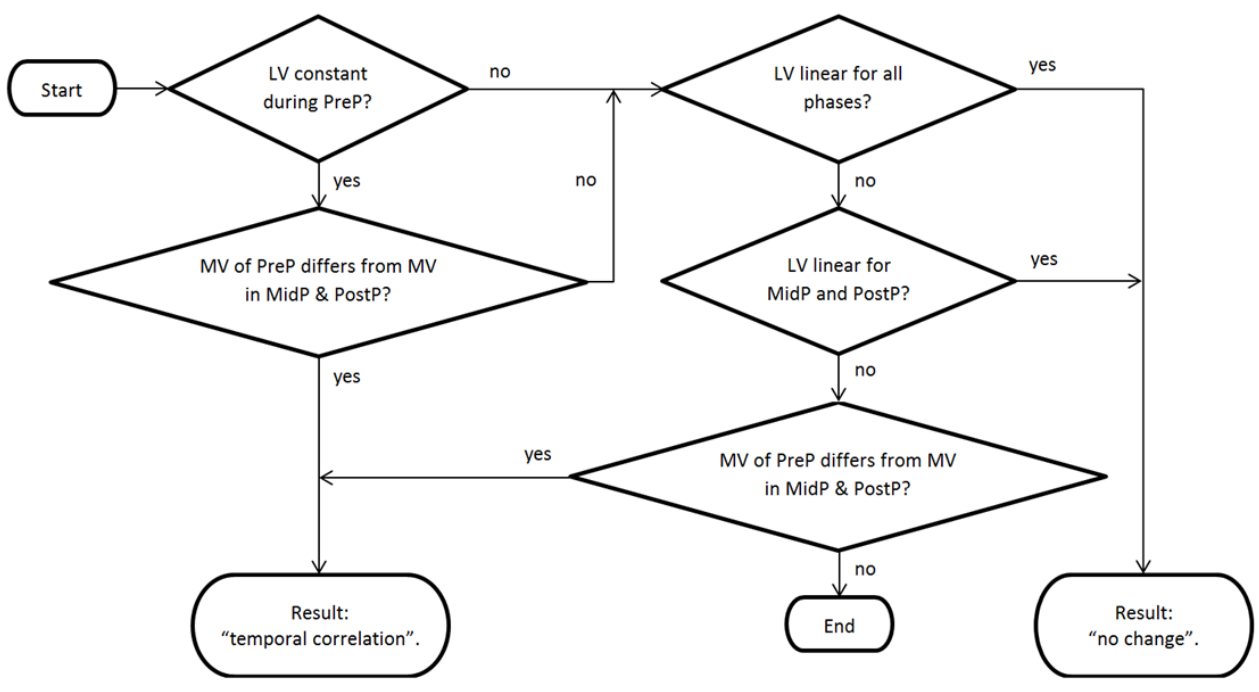

(C)

Post-Processing Flowchart

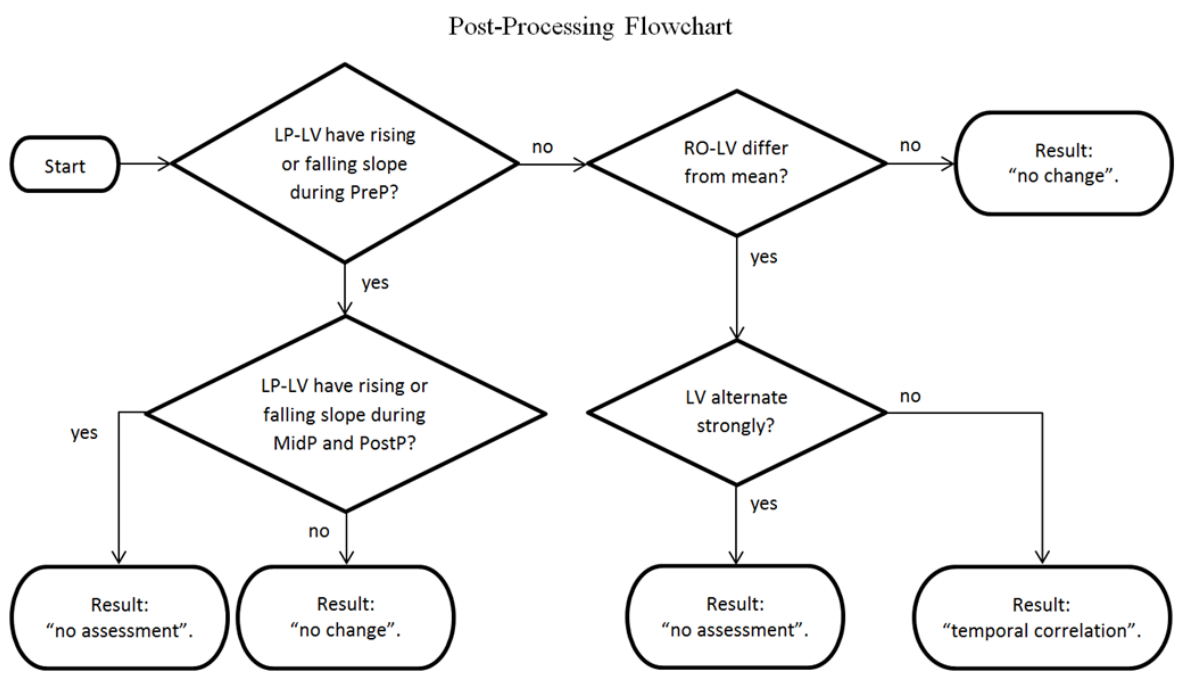

Figure 2: Flowchart of the algorithm. (A) Complete overview; (B) Main Loop; (C) Post-processing Chain. LV: Lab

\section{Algorithm Validation}

As described in the Methods section, the validation of the algorithm was carried out in two steps.

272 First, all curves of the Ground Truth data corpus (Newe et al., 2015) were assessed by the algorithm and the results were then compared to the reference classifications that had been 
274 determined by human experts by means of the Concordance Score $S_{c}$ proposed in (Newe et al., 275 2015). The Concordance Score reached by the algorithm was $S_{c}=0.803$. A more detailed 276 breakdown of the classification results is listed in Table 4 and Table 5 and a mosaic plot is 277 available in Figure 3.

278 Table 4: Classification consensus between ground truth and algorithm output.

\begin{tabular}{|c|c|c|c|}
\hline Algorithm & \multicolumn{3}{|c|}{ Ground Truth } \\
& No Change & Temporal Correlation & No Assessment \\
\hline No Change & 171 & 4 & 2 \\
\hline Temporal Correlation & 40 & 124 & 37 \\
\hline No Assessment & 9 & 5 & 8 \\
\hline
\end{tabular}

\section{Classification Consensus}

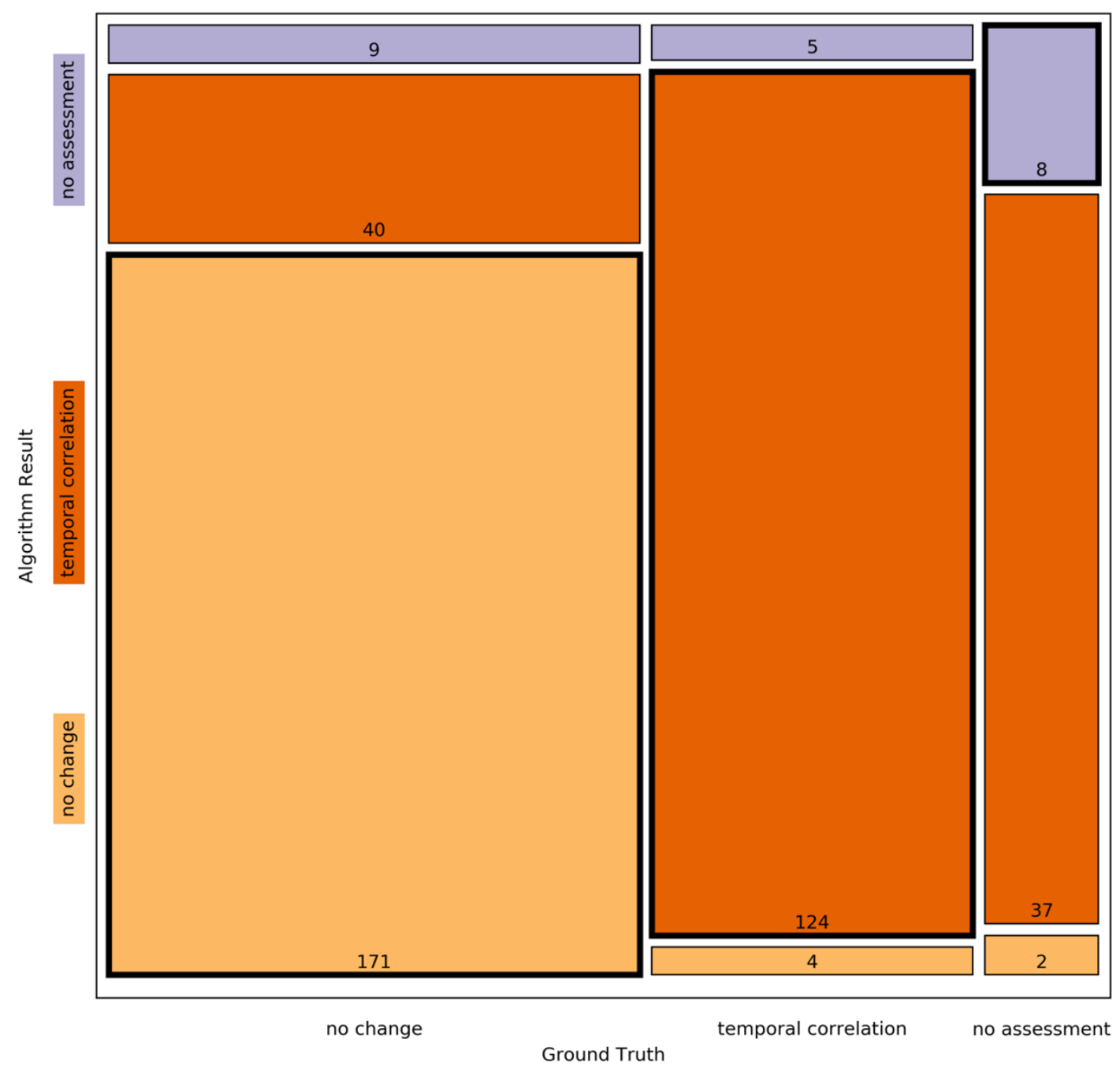

Figure 3: Mosaic plot of classification consensus between ground truth and algorithm output. The width of the faces represents the proportion of the ground truth data; the height represents the proportion of the algorithm result; the figures are the absolute numbers. The boldly framed bars are those in which the algorithm achieved consensus with the ground truth. 
Table 5: Algorithm Sensitivity and Specificity.

\begin{tabular}{|l|l|l|}
\hline Classification & Sensitivity & Specificity \\
\hline No change & 0.777 & $\mathbf{0 . 9 6 7}$ \\
\hline Temporal correlation & $\mathbf{0 . 9 3 2}$ & 0.803 \\
\hline No assessment & 0.170 & 0.960 \\
\hline
\end{tabular}

Second, the processing results of the Ground Truth data corpus were compared in detail to the processing results of a validation dataset. For this purpose, the numbers of classifications found by each step of the algorithm were determined (Table $6 \&$ Figure 4). The difference between both data sets was $\Delta=0.34 \pm 0.30 \%\left(\Delta_{\min }=0.00 \%, \Delta_{\max }=1.07 \%\right)$. Fisher's exact test shows a significant correlation between the classification results of the Ground Truth data and of the validation data $(p=0.9923)$.

Table 6: Detailed comparison of the algorithm results for the Ground Truth data and the validation data. This table lists the absolute (\#) and the relative (\%) numbers of curves in relation to the algorithm step in which they were classified. The last column shows the absolute difference of the relative numbers.

\begin{tabular}{|c|c|c|c|c|c|}
\hline \multirow{2}{*}{$\begin{array}{l}\text { Step Number \& Resulting } \\
\text { Classification }\end{array}$} & \multicolumn{2}{|c|}{ Ground Truth Data } & \multicolumn{2}{|c|}{ Validation Data } & \multirow{2}{*}{$\begin{array}{c}\text { abs. } \Delta \text { of } \\
\%\end{array}$} \\
\hline & $\#$ & $\%$ & $\#$ & $\%$ & \\
\hline $1-$ No Change $^{1}$ & $0^{1}$ & $0.00^{1}$ & $0^{1}$ & $0.00^{1}$ & $n / a^{1}$ \\
\hline 2-Temporal Correlation & 57 & 14.25 & 71 & 14.14 & 0.11 \\
\hline 3-No Change & 126 & 31.50 & 158 & 31.47 & 0.03 \\
\hline 4- No Change & 4 & 1.00 & 6 & 1.20 & 0.20 \\
\hline 5-Temporal Correlation & 51 & 12.75 & 63 & 12.55 & 0.20 \\
\hline 6-Temporal Correlation & 2 & 0.50 & 7 & 1.39 & 0.89 \\
\hline 7 No Change & 20 & 5.00 & 23 & 4.58 & 0.42 \\
\hline 8 - No Change & 11 & 2.75 & 13 & 2.59 & 0.16 \\
\hline 9-Temporal Correlation & 0 & 0.00 & 0 & 0.00 & 0.00 \\
\hline 10 - Temporal Correlation & 2 & 0.50 & 2 & 0.40 & 0.10 \\
\hline 11 - No Change & 0 & 0.00 & 1 & 0.20 & 0.20 \\
\hline 12 - No Change & 0 & 0.00 & 2 & 0.40 & 0.40 \\
\hline 13-Temporal Correlation & 25 & 6.25 & 26 & 5.18 & 1.07 \\
\hline 14 - No Change & 6 & 1.50 & 9 & 1.79 & 0.29 \\
\hline 15 - No Assessment & 20 & 5.00 & 29 & 5.78 & 0.78 \\
\hline 16 - No Change & 10 & 2.50 & 10 & 1.99 & 0.51 \\
\hline 17 - No Assessment & 2 & 0.50 & 1 & 0.20 & 0.30 \\
\hline 18-Temporal Correlation & 64 & 16.00 & 81 & 16.14 & 0.14 \\
\hline
\end{tabular}

${ }^{1}$ The first step ("Preprocessing") is disabled by default and was not considered for the calculation of statistics. 
Classification Analysis

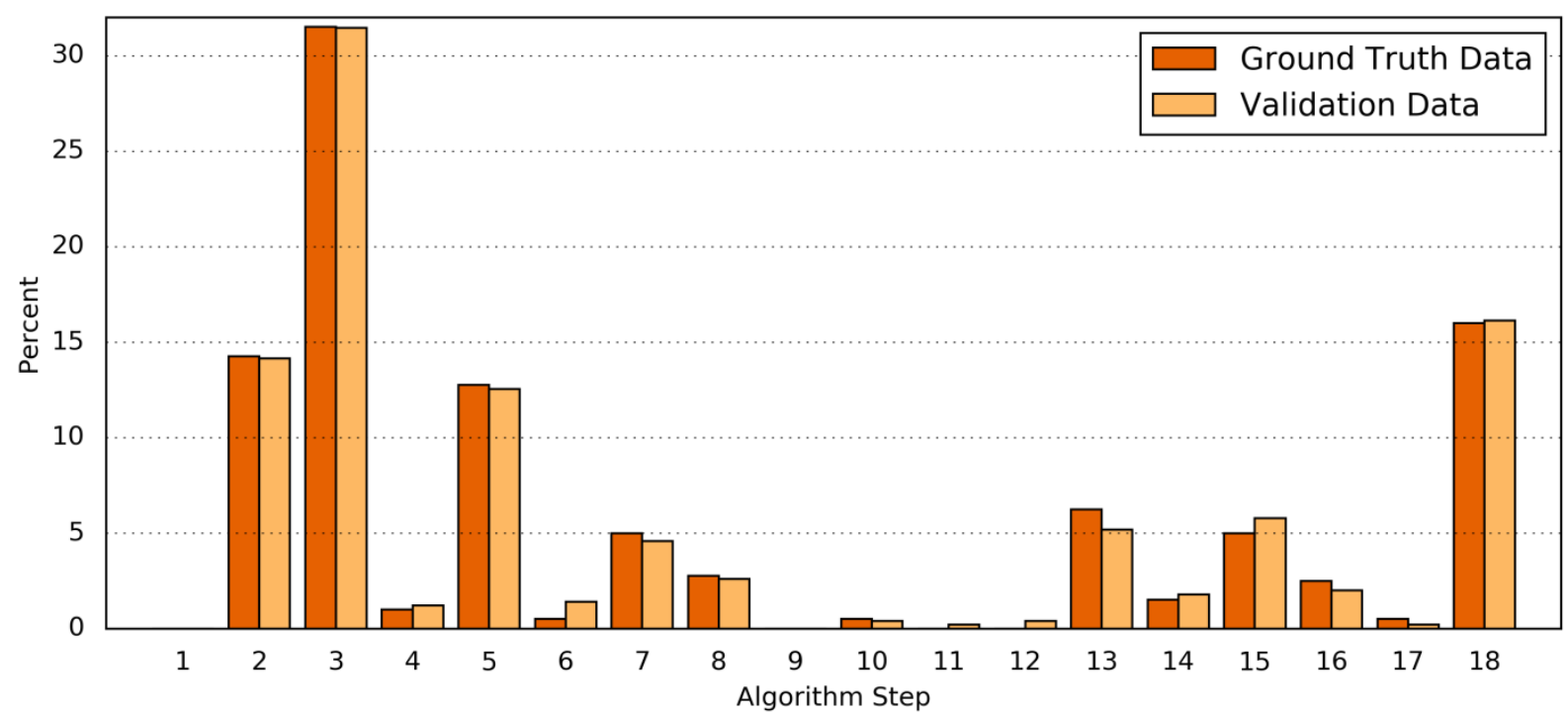

295

296

297

298

299

300

301

302

303

304

305

306

307

308

309

310

311

312

313

314

Figure 4: Comparison of the algorithm steps that yield an assessment result. Plotted are the relative (\%) numbers of curves in relation to the algorithm step in which they were classified for the Ground truth data (green) and the validation data (red). Note that the first step ("Preprocessing") is disabled by default.

\section{Reference Implementation}

The reference implementation of the algorithm is available in Python programming language. It requires the Python packages SciPy (Jones, Oliphant \& Peterson, 2001) version 0.16 .1 and numpy (van der Walt, Colbert \& Varoquaux, 2011) version 1.9 .3 and is available as Supplemental File S1.

As mentioned in the introduction, one of the problems of EHR data analyses is incomplete data. This also applies to the Ground Truth dataset: in some cases, the episodes of lab value observations are interrupted by gaps (e.g., curve \#006). Since most implementations of standard functions in SciPy require continuous values, those gaps are temporarily filled with zeros during the processing, while attributing these artificial values with a very low weighting of $10^{-6}$. As a result, the error introduced by the filling of the gaps becomes negligible. When the weighting was increased for testing purposes, the assessment results started to vary at a value of $10^{-3}$; therefore $10^{-6}$ was considered to be sufficient.

The entry to the reference implementation is the TCAlgo_TestExecution.py file. In line 27, a source XML file can be specified. This source file must match the structure displayed in Figure 5 (the name of the root element can be arbitrary). 


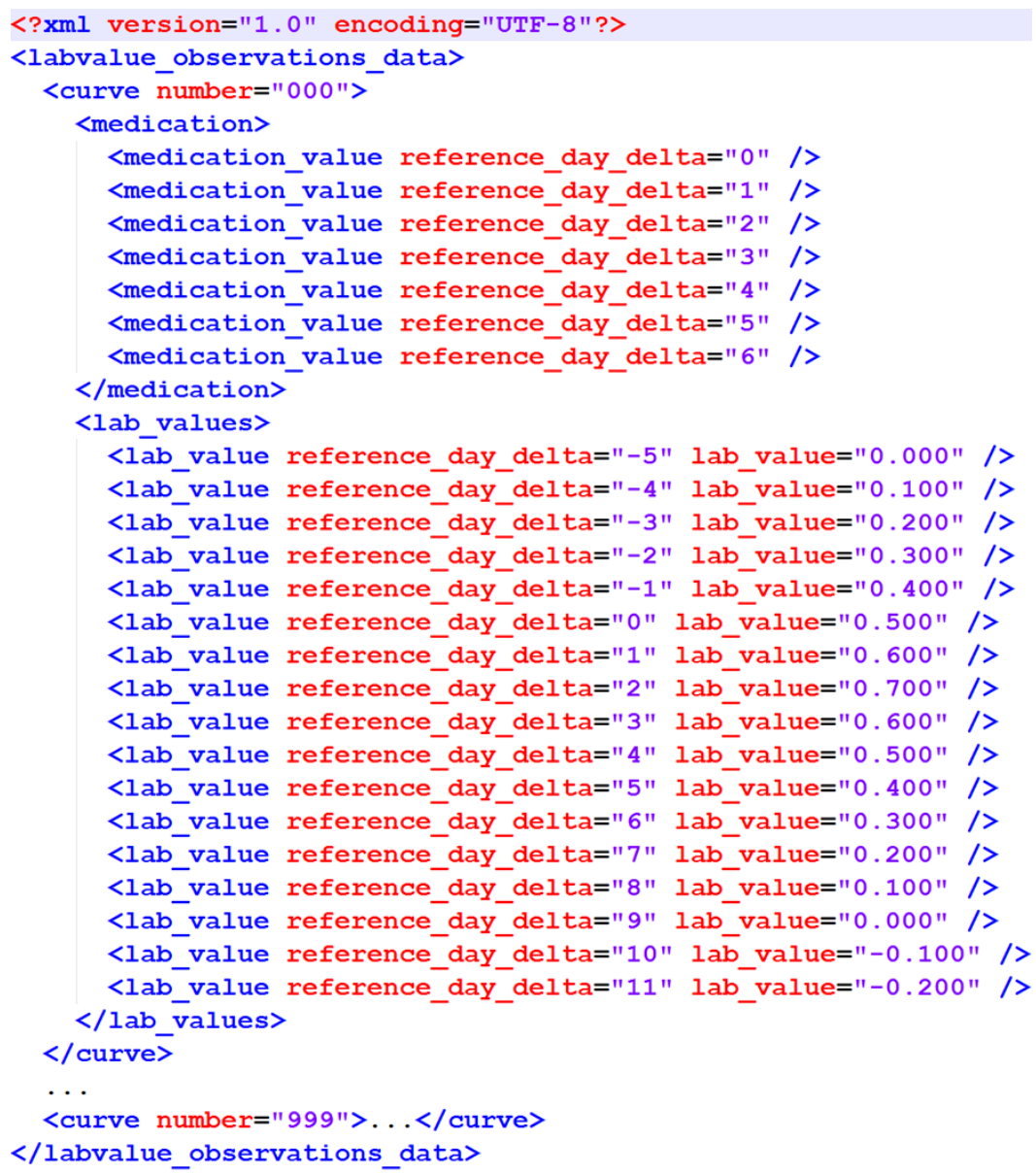

316 Figure 5: XML structure of the input XML. This XML structure is required by the importer of the reference 317 implementation.

In line 30, the main global parameter is set: the noChangeIfInReferenceRange switch can be activated or deactivated here. If it is activated, lab values that only vary within their reference range [0..1] are automatically assessed as "no change" (see description of the optional pre-processing step above). This switch is deactivated by default. details, please follow to the embedded documentation.

326 The assessment result is written to the algorithm_assesssment_result.xml file.

\section{Discussion}


330

331

332

333

334

335

336

337

338

339

340

341

342

343

344

345

346

347

348

349

350

351

352

353

354

355

356

357

358

359

360

361

362

correlations between drug administrations and lab value alterations. The default parameters have been adjusted to match best a ground truth of such correlations published as a result of previous work (Newe et al., 2015). A reference implementation in Python programming language is available as Supplemental File S1.

The algorithm can be used as a means for dramatyping EHR data in order to detect Observable Drug Events (ODEs) in EHRs or similar databases. It is not suitable (and notintended) as a sole means for the detection of ADRs, but it can serve as a module of a greater framework and thus contribute to ADR detection since ODE detection is a precursor of ADR detection. Creating this framework for ADR detection is subject of further research and therefore not within the scope of this article.

For some time, human expertise in the pharmacovigilance domain will certainly be the critical factor regarding ADR detection and ADR identification. However, assistance for these experts can well be provided by data processing tools. Instead of using it only for the identification of signals which are worthy of further investigation - as proposed in (Hauben et al., 2005) -,the algorithm presented in this article can also be used as a screening tool for the exclusion of datasets from further investigation. In doing so, the data that needs to be reviewed by human experts can be reduced significantly (about 44\% in the case of the Ground Truth dataset) with the high specificity of 0.967 for the "no change" classification ensuring reliable results.

Especially the latter aspect should be kept in mind if the parameters are intended to be modified in future iterations or applications of the algorithm. A false-positive "no-change" classification is the worst decision since it would conceal a possible temporal correlation from the review by a human expert. Consequently, the specificity of the "no change" classification should always be kept as high as possible.

\section{Limitations}

It is important to resist the temptation to equate detected temporal correlations with causal relationships, especially as regards ADR detection.

ADR detection is a complex task and the temporal correlation between the change of a lab value course and the admission of a drug is only one component of the criteria for a standardized case causality assessment of ADRs (WHO, 2005). The existence of a temporal correlation is, however, a necessary component. I.e., if no temporal correlation exists, the existence of an ADR can be denied.

As (Harpaz et al., 2010) already pointed out with respect to causality assessment, the issue of confounding is a serious concern, since it may lead to biased inference. Possible confounders 
363

364

365

366

367

368

369

370

371

372

373

374

375

376

377

378

379

380

381

382

383

384

385

386

387

388

389

390

391

392

393

394

are co-medication, co-morbidities, or the underlying disease itself (WHO, 2005; Harpaz et al., 2010). Since the presented algorithm processes normalized data without any information about the laboratory parameters or the drugs, it does not take into account the effect of possible confounders. However, this is intentional and a comprehensive ADR detection is not within the focus of the algorithm.

\section{Validation Methodology and Result}

The objective was to design an algorithm that reproduces the assessment results of human experts whereby one has to consider that applicable data in this field is rare - in fact, (Newe et al., 2015) is the only available and validated source of such data. The algorithm is based on rules that were induced from this data and in order to consider as much of this limited input data as possible (Hawkins, 2004), the Ground Truth data set was not divided into training data and validation data. Instead, the validation was performed in two steps.

First, the rules were verified deductively in order to proof that the inductive reasoning process was valid. The default parameters yield an overall Concordance Score $S_{C}$ of 0.803 which is not a $100 \%$ match, but still within in the range of the Concordance Scores of the original assessors in (Newe et al., 2015) (minimum $S_{C}=0,766$ for Assessor 05; maximum $S_{C}=0,883$ for Assessor 08). Therefore, the overall result achieved by the algorithm is equal to the results of the assessments made by human experts and better than the result of the worst performing human expert.

In order to address the overfitting problem, a validation dataset with the same provenance as the Ground Truth dataset was used (Hawkins, 2004). The algorithms assesses both datasets with nearly the same outcome $(p=0.9923)$. Since both the Ground Truth data and the validation data originate from the same raw data it can be assumed that the algorithm produces valid results without being overfitted to the Ground Truth data set (Hawkins, 2004).

Another aspect that should be considered is, that one single step of the algorithm (\#3) covers more than $30 \%$ of the data and that a total of four steps (\#2, \#3, \#5,\#18) covers nearly $75 \%$ of the data (in both the Ground Truth data and the validation data). The number of steps is much smaller than the number of data points in the Ground Truth dataset $(400: 17 \approx 23: 1)$. This provides further evidence that the algorithm is not biased by overfitting.

\section{Other Approaches of Mining EHRs for Drug Safety Signals}

Several studies and large-scaled projects examined the possibilities to retrospectively detect ADRs on the basis of EHR data. 
395

396

397

398

399

400

401

402

403

404

405

406

407

408

409

410

411

412

413

414

415

416

417

418

419

420

421

422

423

424

Chazard developed and implemented 236 partly very complex rules for the detection of selected ADRs that were not limited to DLVI (Chazard, 2011). (Liu et al., 2012) used a timelinebased approach to correlate drug administrations with possible ADR diagnoses that were extracted from textual medical records by means of Natural Language Processing (NLP). (Rozich, Haraden \& Resar, 2003) evolved a method introduced by (Classen et al., 1991) and used 24 selected triggers (including 12 well-defined lab value conditions) to identify ADRs in a setting of 86 hospitals. (Harpaz et al., 2010) published ab article about the feasibility of a method that has been designed to perform an automatic mining of narrative texts in EHRs for the identification of ADE signals, and, at the same time, taking account of confounding factors.

The EU-ADR project exploited eight EHR databases of four European countries in order to detect drug safety signals (Trifirò et al., 2011; Coloma et al., 2013). The SALUS project, which has been funded with more than $€ 3$ million by the European Union (http://www.salusproject.eu/) is concerned with the creation of a proactive solution for the detection of ADRs based on EHR data. It aims to provide a standard-based interoperability framework in order to enable the performance of safety studies that can analyze real-time patient data in communication with heterogeneous EHR systems. The Sentinel Initiative, governed by the FDA (FDA, 2008), is a comparable project in the United States.

The work presented in this article, by contrast, takes a more generic approach and focuses on ODEs rather than ADRs. In addition, the presented algorithm does not need any information about the involved laboratory parameters or the specific drug(s). Therefore, it can be used not only as a module of a larger framework that is aimed to detect ADRs, but also for other purposes like the assessment of data quality or the verification of desired drug effects.

\section{Conclusion}

In this article, an algorithm for detecting reasonable temporal correlations between drug administration and laboratory value alterations is presented. It processes normalized values and is thus universally applicable. It has a specificity (0.967) for the detection of lab value courses that show no change in temporal correlation with drug administrations and it has a sensitivity (0.932) for the detection of lab value courses that do show a change. Therefore it is very well suited to screen EHRs and to support human experts in the search of ADRs. 
$425 \quad$ List of abbreviations

426 ADE - Adverse Drug Event

427 ADR - Adverse Drug Reaction

428 DLVI - Drug-Lab Value Interaction(s)

429 EHR - Electronic Health Record

430

431

432

433

434

435

436

437

438

FAERS - FDA Adverse Event Reporting System

FDA - U.S. Food and Drug Administration

NLP - Natural Language Processing

ODE - Observable Drug Event

U.S., USA - United States of America

WHO - World Health Organization

$\mathrm{XML}$ - Extensible Markup Language

\section{Acknowledgements}

439 The present work was performed in fulfillment of the requirements for obtaining the degree "Dr. rer. biol. hum."

440 from the Friedrich-Alexander-Universität Erlangen-Nürnberg (FAU). 


\section{References}

BfArM (Bundesinstitut für Arzneimittel und Medizinprodukte (German Federal Institute for Drugs and Medical Devices)). 2015. [Adverse reaction reporting by health professionals]. Available at http://www.bfarm.de/DE/Arzneimittel/Pharmakovigilanz/RisikenMelden/uaw/_node (accessed: 201511-30). Archived at http://www.webcitation.org/6RZz9q3ah.

Chazard E. 2011. Automated Detection of Adverse Drug Events by Data Mining of Electronic Health Records. Available at https://hal.archivesouvertes.fr/docs/00/63/72/54/PDF/these_emmanuel_chazard.pdf (accessed: 2015-11-30). Diss., Université Lille Nord de France.

Classen DC, Pestotnik SL, Evans RS, Burke JP. 1991. Computerized surveillance of adverse drug events in hospital patients. Journal of the American Medical Association, 266(20):2847-2851. DOI: 10.1001/jama.1991.03470200059035. PMID: 15933322.

Classen DC, Resar R, Griffin F, Federico F, Frankel T, Kimmel N, Whittington JC, Frankel A, Seger A, James BC. 2011. 'Global Trigger Tool' Shows That Adverse Events In Hospitals May Be Ten Times Greater Than Previously Measured. Health Affairs, 30(4):581-589. DOI: 10.1377/hlthaff.2011.0190. PMID: 21471476.

Coloma PM, Avillach P, Salvo F, Schuemie MJ, Ferrajolo C, Pariente A, Fourrier-Réglat A, Molokhia M, Patadia V, van der Lei J, Sturkenboom M, Trifiro G. 2013. A Reference Standard for Evaluation of Methods for Drug Safety Signal Detection Using Electronic Healthcare Record Databases. Drug Safety, 36(1):13-23. DOI: 10.1007/s40264-012-0002-x. PMID: 23315292.

Curran WJ. 1971. The thalidomide tragedy in Germany: the end of a historic medicolegal trial. The New England Journal of Medicine, 284(9):481-482. PMID: 5100423.

FDA (U.S. Food and Drug Administration). 2008. FDA's Sentinel Initiative. Available at http://www.fda.gov/Safety/FDAsSentinelinitiative/ucm2007250.htm (accessed: 2015-11-30). Archived at http://www.webcitation.org/6RckFS9zi.

FDA (U.S. Food and Drug Administration). 2014. FDA Adverse Event Reporting System (FAERS). Available at http://www.fda.gov/Drugs/GuidanceComplianceRegulatory/nformation/Surveillance/AdverseDrugEffect s/default.htm (accessed: 2015-11-30). Archived at http://www.webcitation.org/6RZygmjLm.

Grönroos P, Irjala K, Heiskanen J, Torniainen K, Forsström J. 1995. Using computerized individual medication data to detect drug effects on clinical laboratory tests. Scandinavian Journal of Clinical \& Laboratory Investigation, 55(222):31-36. DOI: 10.3109/00365519509088448. PMID: 7569744.

Haddad RA, Akansu AN. 1991. A class of fast Gaussian binomial filters for speech and image processing. IEEE Transactions on Signal Processing, 39(3):723-727. 
476

477

478

479

480

481

482

483

484

485

486

487

488

489

490

491

492

493

494

495

496

497

498

499

500

501

502

503

504

505

506

507

508

Harpaz R, Haerian K, Chase HS, Friedman C. 2010. Mining Electronic Health Records For Adverse Drug Effects Using Regression Based Methods. In Proceedings of the 1st ACM International Health Informatics Symposium; New York. 100-107.

Hauben M, Madigan D, Gerrits CM, Walsh L, Van Puijenbroek EP. 2005. The role of data mining in pharmacovigilance. Expert Opinion on Drug Safety, 4(5):929-948. DOI: 10.1517/14740338.4.5.929. PMID: 16111454.

Hawkins DM. 2004. The Problem of Overfitting. Journal of Chemical Information and Computer Sciences, 44(1):1-12. DOI: 10.1021/ci0342472. PMID: 14741005.

Hersh WR, Weiner MG, Embi PJ, Logan JR, Payne PRO, Bernstam EV, Lehmann HP, Hripcsak G, Hartzog TH, Cimino JJ, Saltz JH. 2013. Caveats for the Use of Operational Electronic Health Record Data in Comparative Effectiveness Research. Medical Care, 51(8):30-37. DOI: 10.1097/MLR.0b013e31829b1dbd. PMID: 23774517.

Hripcsak G, Albers DJ. 2013. Next-generation phenotyping of electronic health records. Journal of the American Medical Informatics Association, 20(1):117-121. DOI: 10.1136/amiajnl-2012-001145. PMID: 22955496.

Hug BL, Keohane C, Seger DL, Yoon C, Bates DW. 2012. The Costs of Adverse Drug Events in Community Hospitals. Joint Commission Journal on Quality and Patient Safety, 38(3):120-126. PMID: 2243522.

Jones E, Oliphant T, Peterson PEA. 2001. SciPy: Open Source Scientific Tools for Python. Available at http://www.scipy.org/ (accessed: 2015-11-30).

Kramer MS. 1981. Difficulties in assessing the adverse effects of drugs. British Journal of Clinical Pharmacology, 11(S1):105S-110S. PMID: 7052788.

Liu Y, LePendu P, Iyer S, Shah NH. 2012. Using Temporal Patterns in Medical Records to Discern Adverse Drug Events from Indications. In AMIA Summits on Trans/ational Science proceedings. 47-56.

Lucas LM, Colley CA. 1992. Recognizing and Reporting Adverse Drug Reactions. Western Journal of Medicine, 156(2):172-175. PMID: 1536067.

Meier F, Maas R, Sonst A, Patapovas A, Müller F, Plank-Kiegele B, Pfistermeister B, Schöffski O, Bürkle T, Dormann H. 2015. Adverse drug events in patients admitted to an emergency department: an analysis of direct costs. Pharmacoepidemiology and Drug Safety, 24(2):176-186. DOI: 10.1002/pds.3663. PMID: 24934134.

Moré JJ. The Levenberg-Marquardt algorithm: implementation and theory. In Lecture Notes in Mathematics. Edited by Watson. Berlin Heidelberg: Springer; 1978:105-116.

Neubert A, Dormann H, Prokosch HU, Bürkle T, Rascher W, Sojer R, Brune K, Criegee-Rieck M. 2013. Epharmacovigilance: development and implementation of a computable knowledge base to identify 
509

510

511

512

513

514

515

516

517

518

519

520

521

522

523

524

525

526

527

528

529

530

531

532

533

534

535

536

537

538

539

540

541

542

adverse drug reactions. British Journal of Clinical Pharmacology, 76(1):69-77. DOI: 10.1111/bcp.12127. PMID: 23586589.

Newe A, Wimmer S, Neubert A, Becker L, Prokosch HU, Beckmann MW, Fietkau R, Forster C, Neurath M. 2015. Towards a Computable Data Corpus of Temporal Correlations between Drug Administration and Lab Value Changes. PLOS ONE, 10(8):e0136131. DOI: 10.1371/journal.pone.0136131. PMID: 26301507.

Pintor-Mármol A, Baena MI, Fajardo PC, Sabater-Hernández D, Sáez-Benito L, García-Cárdenas MV, Fikri-Benbrahim N, Azpilicueta I, Faus MJ. 2012. Terms used in patient safety related to medication: a literature review. Pharmacoepidemiology and Drug Safety, 21(8):799-809. DOI: 10.1002/pds.3296. PMID: 22678709.

Rajakannan T, Mallayasamy S, Guddattu V, Kamath A, Vilakkthala R, Rao PGM, Bairy LK. 2012. Cost of Adverse Drug Reactions in a South Indian Tertiary Care Teaching Hospital. Journal of Clinical Pharmacology, 52(4):599-565. DOI: 10.1177/0091270011398867. PMID: 21505086.

Ramirez E, Carcas A, Borobia A, Lei S, Piñana E, Fudio S, Frias J. 2010. A Pharmacovigilance Program From Laboratory Signals for the Detection and Reporting of Serious Adverse Drug Reactions in Hospitalized Patients. Clinical Pharmacology \& Therapeutics, 87(1):74-86. DOI: 10.1038/clpt.2009.185. PMID: 19890254.

Rozich JD, Haraden CR, Resar RK. 2003. Adverse drug event trigger tool: a practical methodology for measuring medication related harm. Quality and Safety in Health Care, 12(3):194-200. PMID: 12792009.

Russell WMS, Burch RL. The Sources of Physiological Variance. In The principles of humane experimental technique. London: Methuen; 1959.

Siest G. Drug Effects on Laboratory Test Results. Springer; 1980.

Singh HP, Hebert MA, Gault MH. 1972. Effect of some drugs on clinical laboratory values as determined by the Technicon SMA 12/60. Clinical Chemistry, 18(2):137-144. PMID: 5008521.

Sonntag O, Scholer A. 2001. Drug interference in clinical chemistry: recommendation of drugs and their concentrations to be used in drug interference studies. Annals of Clinical Biochemistry, 38(4):376-385. PMID: 11471880.

Stausberg J. 2014. International prevalence of adverse drug events in hospitals: an analysis of routine data from England, Germany, and the USA. BMC Health Services Research, 14(125). DOI: 10.1186/14726963-14-125. PMID: 24620750.

Tatonetti NP, Denny JC, Murphy SN, Fernald GH, Krishnan G, Castro V, Yue P, Tsau PS, Kohane I, Roden DM, Altman RB. 2011. Detecting drug interactions from adverse-event reports: interaction between paroxetine and pravastatin increases blood glucose levels. Clinical Pharmacology \& Therapeutics, 90(1):133-142. DOI: 10.1038/clpt.2011.83. PMID: 21613990. 
543 Trifirò G, Patadia V, Schuemie MJ, M. CP, Gini R, Herings R, Hippisley-Cox J, Mazzaglia G, Giaquinto C, 544 Lorenza S, Pedersen L, Avillach P, Sturkenboom MCJM, Lei VD. 2011. EU-ADR Healthcare Database

545 Network vs. Spontaneous Reporting System Database: Preliminary Comparison of Signal Detection.

546 Studies in Health Technology and Informatics, 166:25-30. DOI: 10.3233/978-1-60750-740-6-25. PMID:

54721685607.

548 van der Walt S, Colbert SC, Varoquaux G. 2011. The NumPy Array: A Structure for Efficient Numerical 549 Computation. Computing in Science \& Engineering, 13(2):22-30.

550 WHO (World Health Organization). 2005. The use of the WHO-UMC system for standardised case 551 causality assessment. Available at

552 http://www.who.int/medicines/areas/quality_safety/safety_efficacy/WHOcausality_assessment.pdf

553 (accessed: 2015-11-30).

554 WHO (World Health Organization). 2008. Fact sheet No 293. Medicines: Safety of medicines - adverse 555 drug reactions. Available at http://www. wiredhealthresources.net/resources/NA/WHO-

556 FS_MedicinesSafetyAdverseReactions.pdf (accessed: 2015-11-30). Archived at

557 http://www.webcitation.org/6RZoymPDQ.

558 WHO (World Health Organization). 2013. World Health Statistics 2013. Available at

559 http://www.who.int/iris/bitstream/10665/81965/1/9789241564588_eng.pdf (accessed: 2015-11-30).

560 ISBN 978-9241564588. Archived at http://www.webcitation.org/6RZrDd805.

561 Young DS, Thomas D, Friedman R, Perstaner L. 1972. Effects of Drugs on Clinical Laboratory Tests.

562 Clinical Chemistry, 18(10):1041-1303. PMID: 4561120.

563 Young DS. Effects of Drugs on Clinical Laboratory Tests. 5th ed. Vol Volume Two: Listing By Drug. Amer 564 Assn for Clinical Chemistry; 2000.

565 Yu K, Nation R, Dooley M. 2005. Multiplicity of medication safety terms, definitions and functional 566 meanings: when is enough enough? Quality and Safety in Health Care, 14(5):358-363. PMID: 16195570. 